\title{
Interface-Dependent Effective Mobility in Graphene Field-Effect Transistors
}

\author{
PATRIK AHLBERG,${ }^{1}$ MALKOLM HINNEMO $\left({ }^{1},{ }^{1}\right.$ SHI-LI ZHANG,${ }^{1}$ and \\ JÖRGEN OLSSON ${ }^{1,2}$
}

\begin{abstract}
1.-Uppsala Universitet Teknisk-naturvetenskapliga fakulteten, Uppsala, Sweden. 2.-e-mail:
\end{abstract} jorgen.olsson@angstrom.uu.se

\begin{abstract}
By pretreating the substrate of a graphene field-effect transistor (G-FET), a stable unipolar transfer characteristic, instead of the typical V-shape ambipolar behavior, has been demonstrated. This behavior is achieved through functionalization of the $\mathrm{SiO}_{2} / \mathrm{Si}$ substrate that changes the $\mathrm{SiO}_{2}$ surface from hydrophilic to hydrophobic, in combination with postdeposition of an $\mathrm{Al}_{2} \mathrm{O}_{3}$ film by atomic layer deposition (ALD). Consequently, the back-gated G-FET is found to have increased apparent hole mobility and suppressed apparent electron mobility. Furthermore, with addition of a top-gate electrode, the G-FET is in a double-gate configuration with independent top- or backgate control. The observed difference in mobility is shown to also be dependent on the top-gate bias, with more pronounced effect at higher electric field. Thus, the combination of top and bottom gates allows control of the G-FET's electron and hole mobilities, i.e., of the transfer behavior. Based on these observations, it is proposed that polar ligands are introduced during the ALD step and, depending on their polarization, result in an apparent increase of the effective hole mobility and an apparent suppressed effective electron mobility.
\end{abstract}

Key words: Double-gate FETs, graphene, surface engineering, ALD

\section{INTRODUCTION}

The lack of a proper bandgap in graphene has prevented its serious consideration for use in fieldeffect devices so far. However, this does not mean that the electrical properties of graphene are of less interest or could not be used under other circumstances. The graphene-based field-effect transistor (G-FET) was one of the first devices to be manufactured in the original study in $2004 .{ }^{1}$ The G-FET is a suitable platform for evaluation and comparison of electronic behaviors, processes, and different methods or dopants. ${ }^{2,3}$ However, there is still much left to understand regarding how interfacial defects affect the transfer behavior of the standard G-FET. Lacking a bandgap, graphene is often combined with other chemicals to affect its transfer behavior. These types of chemical interference can readily

(Received July 6, 2017; accepted December 12, 2017 ; published online January 4, 2018) introduce defects or interface charges. If these phenomena are not properly understood, the effect of a process may be misinterpreted.

One specific category of interfacial additives are those of polar nature. Such additives have been shown to have a great effect on the mobility of the G-FET when in the vicinity of the graphene. ${ }^{4}$ In previous studies, such chemical treatment of graphene led to vast differences between hole and electron conductance. ${ }^{4,5}$

The ratio of the slope, i.e., transconductance, on the electron and hole side of the transfer curve is normally reported to be close to $1{ }^{6}$ However, this article will show that this value can be pushed significantly higher, simply by making the surface hydrophobic. It is shown that this effect arises from changing the conditions for $\mathrm{Al}_{2} \mathrm{O}_{3}$ deposition (Fig. 1). This effect is of great interest, as transfer curves are commonly used to estimate the mobility, and not taking this effect into account could thus lead to wrong conclusions. The method of using 


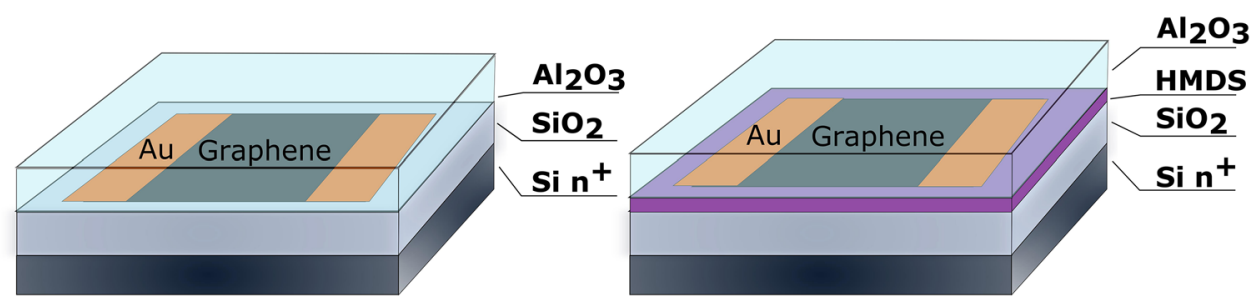

Fig. 1. G-FET stack with and without HMDS treatment before graphene transfer. Graphene with Au contacts shown in the center of each sketch.

hydrophobic substrates to minimize the effect of surrounding humidity has been suggested as a step toward reliable G-FET production. ${ }^{7}$ The data presented here suggest that this is not a straightforward strategy.

It has previously been shown that atomic layer deposition (ALD) of $\mathrm{Al}_{2} \mathrm{O}_{3}$ can deliver high-quality, apparently nondoped graphene devices. ${ }^{8}$ Graphene is inherently hydrophobic, ${ }^{9}$ and the ALD process, which combines water and trimethylaluminum (TMA), can be problematic to implement. It is also known that, even though graphene is hydrophobic, it is thin enough that it can, to a certain extent, borrow the wettability of the surface below it. ${ }^{9}$ For hydrophilic substrates such as $\mathrm{SiO}_{2}$, the problem of depositing materials on graphene by ALD can be circumvented by covering the surface with a seed layer prior to deposition, to help initialize the growth. ${ }^{10}$ However, introducing bis(trimethylsilyl)amine (HMDS), a known surfactant for increasing the hydrophobicity of the $\mathrm{SiO}_{2}$ surface, prior to transfer of the graphene will greatly affect the ability of the ALD process to deposit a high-quality film of $\mathrm{Al}_{2} \mathrm{O}_{3}$. This change of the substrate wettability is here shown to alter the $\mathrm{Al}_{2} \mathrm{O}_{3}$ film properties and thereby efficiently affect the ambipolar behavior of the G-FET and increase the effective hole mobility.

G-FETs are studied both without a top gate (showing the most pronounced elevation/suppression of mobility) and with a top gate covering the channel.

\section{EXPERIMENTAL PROCEDURES}

Graphene was produced by chemical vapor deposition (CVD) on $\mathrm{Cu}$-foil substrate with $\mathrm{Ar}: \mathrm{H}_{2}: \mathrm{CH}_{4}$ feed gases mixed at ratio of 100:75:1. After the CVD process, graphene/Cu was covered in poly(methyl methacrylate), and a clean low-pressure transfer process was performed onto a highly doped Si wafer covered by 340 -nm thermally grown oxide on top. ${ }^{11}$ The $\mathrm{SiO}_{2} / \mathrm{Si}$ substrate was patterned with $\mathrm{Pd} / \mathrm{Ti}$ contact pads using lift-off and primed with HMDS prior to graphene transfer. With graphene in place on the wafer, 80-nm Au film was evaporated and test structures manufactured in accordance with our previously reported method. ${ }^{8}$ The top dielectric was $\mathrm{Al}_{2} \mathrm{O}_{3}$, deposited by means of ALD, combined with a 1-nm Al seed layer, to thickness of $56 \mathrm{~nm}$
(600 cycles) to avoid pinholes and top-gate leakage. The ALD feed gases were TMA and water. The ALD process was divided into two sets of 300 cycles to

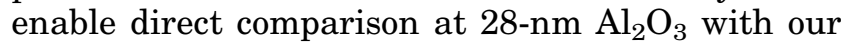
previously published G-FET. The top gate was added by evaporation of $\mathrm{Cu}$ and defined by photolithography and wet etching. The G-FETs were characterized electrically using a probe station with an HP4155A parameter analyzer for direct-current (DC) measurements.

\section{RESULTS AND DISCUSSION}

It was noticed that, of the G-FETs that were conducting prior to the first 300 ALD cycles, only those with channel width $(W)$ of $500 \mu \mathrm{m}$ were still operational afterwards. Structures with smaller width were no longer conducting, and no wider channels were present in the design. This effect, i.e., that small devices did not survive, likely results from the complex wetting behavior of the surface. All the 500- $\mu \mathrm{m}$-wide devices, regardless of length $(L)$, were functioning and exhibited repeatable behavior. This means that the effective channel width could be below $500 \mu \mathrm{m}$. The transfer curves are also depicted in this work as a function of gate field to enable comparison between different dielectrics. After deposition of $28-\mathrm{nm} \mathrm{Al}_{2} \mathrm{O}_{3}$ by $\mathrm{ALD}$, but before deposition of the top gate, the transistors showed clear unipolar behavior with on/off ratio of $\sim 6$ (Fig. 2b). This is in stark contrast to the standard behavior of a conventional G-FET, which is commonly symmetrical around the minimum conductivity voltage, the Dirac voltage $\left(V_{\text {Dirac }}\right)$, while the effective hole and electron mobility are less than a factor 2 from each other (Fig. 2b, inset).

The Raman spectrum collected after transfer confirmed that the graphene was indeed single layer (Fig. 2a). After the first $28 \mathrm{~nm}$ of ALD, $V_{\text {Dirac }}$ shifted to $130 \mathrm{~V}$ (corresponding to electric field of $3.8 \times 10^{6} \mathrm{~V} / \mathrm{cm}$ ), measured at drain-source voltage of $V_{\mathrm{DS}}=100 \mathrm{mV}$, as compared with the reference device (Fig. 2c). This doping-induced shift in $V_{\text {Dirac }}$ is according to $C_{\mathrm{ox}}=q N / V_{\text {Dirac }}$, correlated to hole doping concentration of $8.2 \times 10^{12} \mathrm{~cm}^{-2} . C_{\text {ox }}$ is the capacitance of the dielectric oxide, $N$ is the doping concentration, and $q$ is the elementary charge. The second deposition of ALD did not affect the behavior of the transistors any further. 

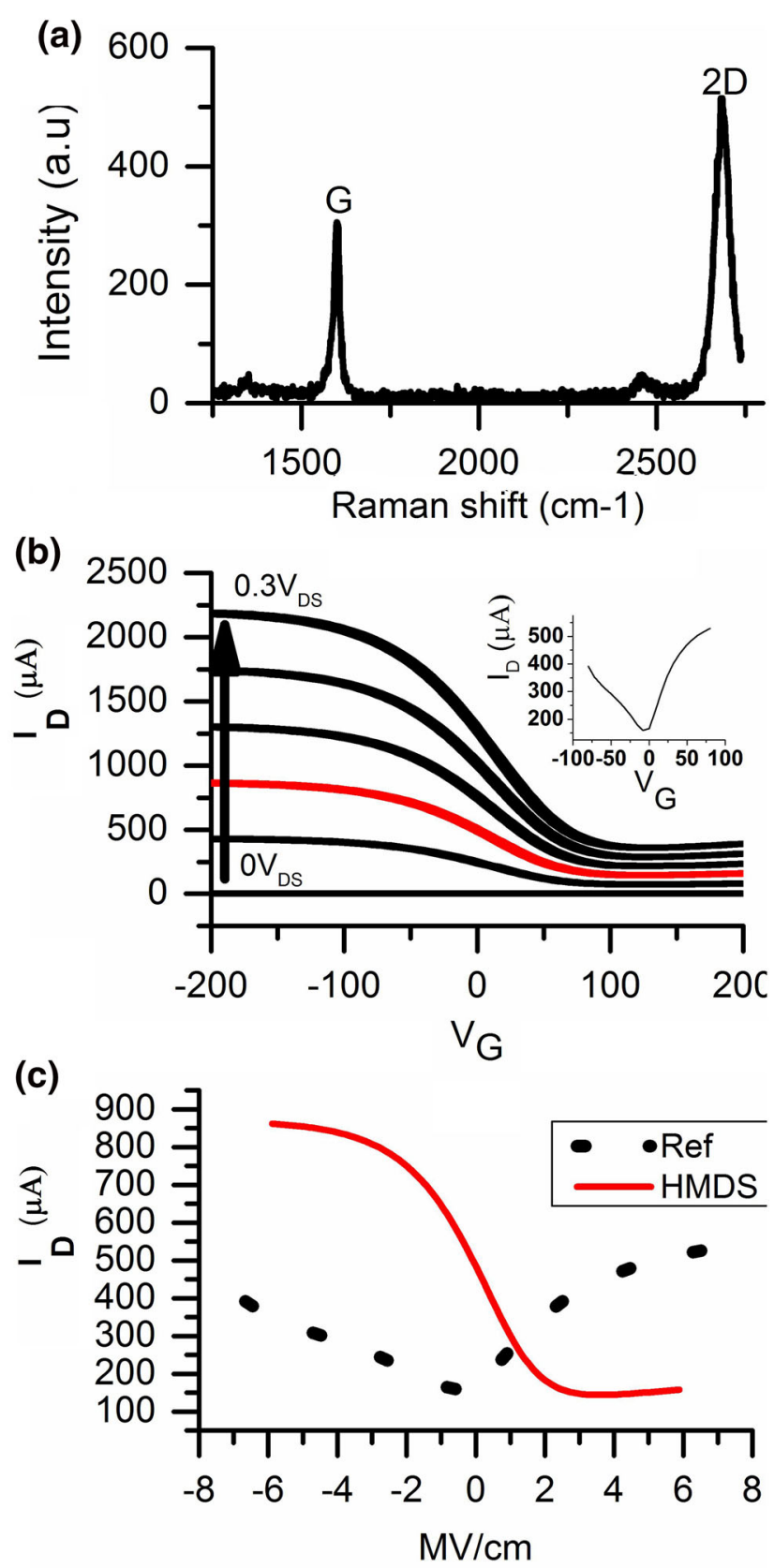

Fig. 2. (a) Representative Raman spectrum of graphene used: the $G$ and $2 \mathrm{D}$ peaks are marked. (b) Drain current versus back-gate voltage of a G-FET on HMDS-treated $\mathrm{SiO}_{2}$ with 28-nm $\mathrm{Al}_{2} \mathrm{O}_{3}$ from ALD, $V_{\mathrm{DS}}$ is stepped from $0 \mathrm{~V}$ to $0.3 \mathrm{~V}$, with the arrow indicating the direction ( $L=50 \mathrm{um}$ ). Inset: Transfer curve of an untreated G-FET as in previous work $\left(V_{\mathrm{DS}}=0.1 \mathrm{~V}\right) .^{8}$ (c) Drain current versus backgate field for two of the curves in (b). The untreated G-FET corresponds to the inset in (b) ( $V_{\mathrm{DS}}=0.1 \mathrm{~V}$, dotted curve) while the HMDS-treated G-FET corresponds to the red curve in (b) $\left(V_{\mathrm{DS}}=0.12 \mathrm{~V}\right.$, red curve) (Color figure online).

After the top-gate electrode was introduced, the unipolar behavior disappeared and $V_{\text {Dirac }}$ shifted to $-12 \mathrm{~V}$ (corresponding to electric field of $\left.-3.5 \times 10^{5} \mathrm{~V} / \mathrm{cm}\right)$, which correlates with an electron density level of $7.5 \times 10^{11} \mathrm{~cm}^{2}$, measured with the top gate grounded. For the same transistor, a floating top gate resulted in an even more negative shift of $V_{\text {Dirac }}$, likely due to capacitive coupling from the back gate to the top gate. ${ }^{12}$

However, when the top gate was set to a negative potential, unipolar behavior reemerged. In fact, the transconductance $\left(g_{\mathrm{m}}\right)$, which is proportional to the mobility, was found to change nonlinearly as the top-gate potential was stepped to lower voltages (Fig. 3a). The maximum and minimum transconductance both decreased with decreasing top-gate bias, while at the same time the distance between these extreme values increased. As these extreme values are dependent on the mobility of the G-FET, this means that the apparent hole mobility, linked to the lowest (negative) transconductance, increases, while its electron counterpart, linked to the maximum transconductance, decreases. As the mobilities evolve at different paces, the result is a more unipolar behavior. This effect was only found when the top gate was stepwise controlled and the back gate was swept. No such obvious effect could be measured when the back gate was stepwise controlled and the top gate was swept (Fig. 3b). This suggests that the unipolar behavior is somehow connected to the $\mathrm{Al}_{2} \mathrm{O}_{3}$ being at a negative potential. It has been shown in several publications that ALD of $\mathrm{Al}_{2} \mathrm{O}_{3}$, when not deposited correctly, can result in a significant amount of trapped negative charge, resulting in a $p$-doping effect on the affected transistor. ${ }^{13,14}$ When the top-gate metal was added, it balanced the intrinsic charge in the $\mathrm{Al}_{2} \mathrm{O}_{3}$ and allowed the G-FET to act as if the charge had been removed.

Similar behavior (Fig. 2c) has been previously published, where the graphene was under the influence of dipole molecules or redox couples, i.e., $\mathrm{H}_{2} \mathrm{O}$ or $\mathrm{H}_{2} \mathrm{O} / \mathrm{O}_{2} \cdot{ }^{4,15}$ However, the influence of HMDS on the wettability of graphene makes the presence of water on the graphene surface highly unlikely. ${ }^{16}$ The ALD process relies on water being able to wet the surface so that it will react with the subsequent TMA. Due to the low wettability of this graphene, it is likely that there are unreacted ligands in the $\mathrm{Al}_{2} \mathrm{O}_{3}$ at the graphene interface, i.e., methyl and hydroxyl groups. These ligands would have been protected from reacting and therefore remain after the ALD process had finished. These Al-ligand groups can, especially when oriented in an electric field, adopt a dipole behavior; i.e., the ligands align with the negative field through the $\mathrm{Al}_{2} \mathrm{O}_{3}$. In this case, the ligands presumably have negative net charge close to the graphene interface. Assuming this negative net charge to be independent of the back-gate potential, different situations can occur, as shown in Fig. 3c; i.e., at negative backgate potential, holes in the graphene may scatter less due to a balanced charge situation, leading to increased hole mobility, while for positive back-gate potential, the field effect for electrons increases their scattering, thus decreasing the electron 

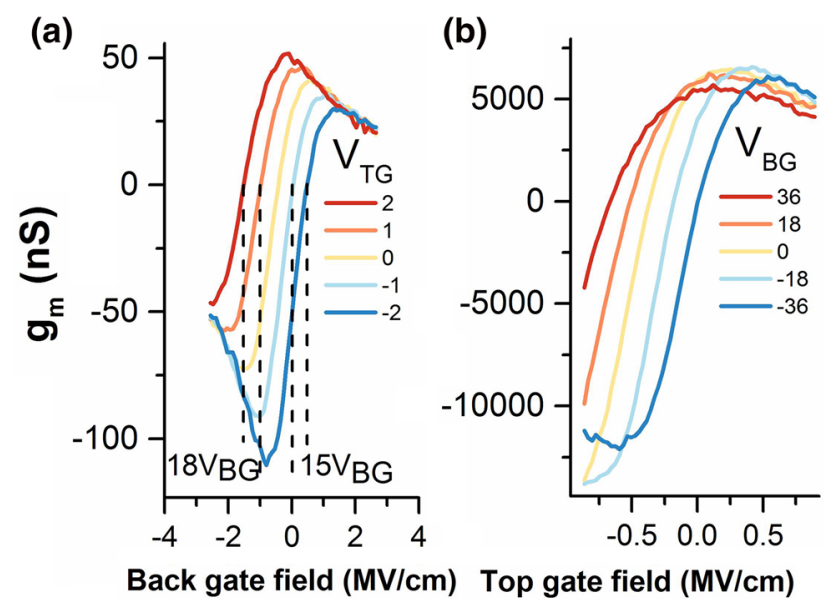

(c)

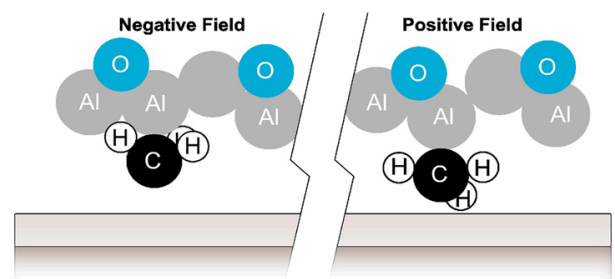

Fig. 3. Transconductance of a G-FET: (a) for different top-gate potentials when sweeping the back gate potential, and (b) for different back-gate potentials when sweeping the top-gate potential. (c) Sketch of a negative (left) or positive (right) field over the $\mathrm{Al}_{2} \mathrm{O}_{3}$ and how that would align a methyl ligand.

mobility. However, in the presence of a top gate, its potential now controls the electric field through the oxide, thus determining the ligand orientation.

To rule out that this effect did not simply originate from mobile charges or oxide trapped charges, a study was performed, altering the delay time between measured data points. With the presence of mobile charges or oxide trapped charges, there should be a significant increase of the hysteresis and decrease of the effective mobilities in the transfer characteristics as the delay time is increased. However, it was observed that the delay time had only a minimal effect on the transfer curves. The data in this paper were collected at $0 \mathrm{~s}$ delay time, but even when held for $10 \mathrm{~s}$ per data point, only a slight increase of the hysteresis was observed.

It was also noted that, as the top-gate potential was changed linearly, $V_{\text {Dirac }}$ did not change in the same way (Fig. 3a). For a conventional G-FET, the transfer curve is the result of the sum of the different electric fields over the channel. This means that a linear change of the top-gate potential is expected to result in a linear shift of $V_{\text {Dirac }}$, proportional to the dielectric thickness. ${ }^{8}$ However, as the top-gate potential decreases in these G-FETs, the effect it has on the transfer curve diminishes measurably. The shift of the Dirac voltage becomes smaller for each linear step of the top gate. With step length of $1 \mathrm{~V}$ for the top-gate potential $\left(V_{\mathrm{TG}}\right)$,

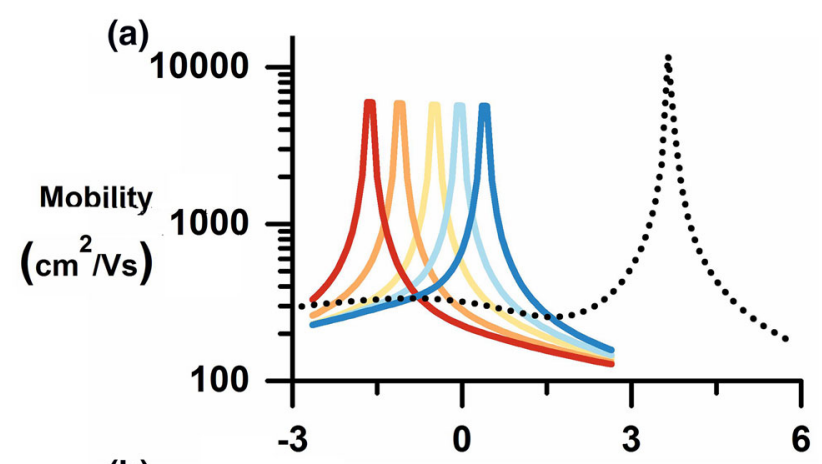

(b)

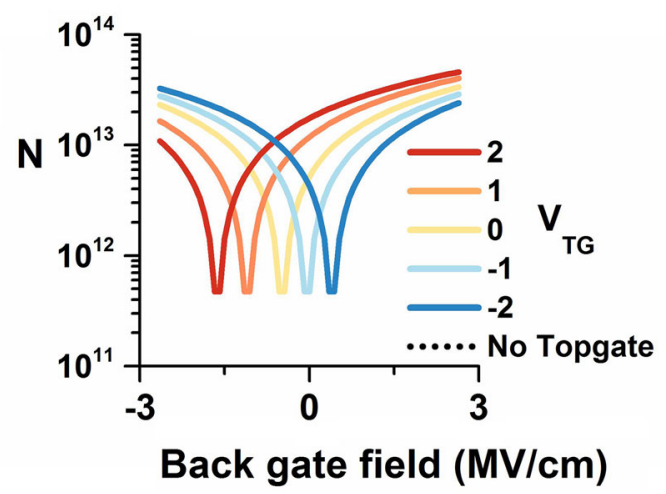

Fig. 4. (a) Effective mobility for G-FET at different top-gate voltages, calculated from the data in Fig. $3 a$, as well as the device without a top gate (dotted curve), calculated from the data in Fig. 2c. $V_{\text {Dirac }}$ has been rounded off to fit in the middle of two data points to yield comparative curves. (b) The carrier concentration corresponding to the mobilities in (a).

$V_{\text {Dirac }}$ shifts from $18 \mathrm{~V}$ between $V_{\mathrm{TG}}=1 \mathrm{~V}$ and $V_{\mathrm{TG}}=2 \mathrm{~V}$ to $15 \mathrm{~V}$ between $V_{\mathrm{TG}}=-2 \mathrm{~V}$ and $V_{\mathrm{TG}}=-1 \mathrm{~V}$ (Fig. 3a). This effect supports the previously stated mechanism of dipole ligands orienting on the graphene surface.

The channel conductance $\left(g_{\mathrm{D}}\right)$ is defined as $\delta I_{\mathrm{D}} / \delta V_{\mathrm{D}}$, here being approximated as $I_{\mathrm{D}} / V_{\mathrm{DS}}$, where $V_{\mathrm{DS}}=10 \mathrm{mV}$. This approximation is possible as the graphene channel conductance is measured to be linear in the drain voltage range of $-1 \mathrm{~V}$ to $1 \mathrm{~V}$. The channel charge $\left(Q_{\mathrm{n}}\right)$ is estimated from $Q_{\mathrm{n}}=C_{\mathrm{ox}}\left(V_{\mathrm{G}}-V_{\text {Dirac }}\right){ }^{10}$ where $V_{\mathrm{G}}$ is either the top-gate potential or the back-gate potential $\left(V_{\mathrm{BG}}\right)$. This estimation is applicable since the quantum capacitance of graphene in this structure is more than $10^{3}$ times larger than the oxide capacitance $C_{\text {ox }}$, and can therefore be neglected. From this approximation, the effective mobility $\left(\mu_{\mathrm{eff}}\right)$ was calculated using the equation ${ }^{10}$

$$
\mu_{\mathrm{eff}}=\frac{g_{\mathrm{D}} L}{W Q_{\mathrm{n}}} .
$$

The effective mobility for all top-gate voltages was observed to decrease, as a function of back-gate field, asymptotically towards about $230 \mathrm{~cm}^{2} / \mathrm{V}$-s for holes and $130 \mathrm{~cm}^{2} / \mathrm{V}$-s for electrons (Fig. 4a). However, when extracting the effective mobility of the 
transistor without the top gate (Fig. 2c, red curve), the mobility behavior was quite different. The effective hole mobility increased to a peak value of $340 \mathrm{~cm}^{2} / \mathrm{V}$-s in the linear region before saturating at around $280 \mathrm{~cm}^{2} / \mathrm{Vs}$, whereas the effective electron mobility decreased toward $170 \mathrm{~cm}^{2} / \mathrm{V}$-s without saturating. As the effective width of the transistors could be different from the nominal value $(500 \mu \mathrm{m})$, the exact numbers could be different. However, the width should be the same for all components, and most importantly, the trend should be the same. The behavior of the hole mobility supports the presence of an enhancement effect for negative fields.

From the effective mobility, the carrier concentration $(N)$ can be calculated using Eq. $2 .^{10}$ The minimum carrier concentration with the top gate present was calculated to be $\sim 4.7 \times 10^{11} \mathrm{~cm}^{-2}$, independent of the top-gate field (Fig. 4b), which corresponds well to the theoretical minimum of $\sim 9 \times 10^{10} \mathrm{~cm}^{-2}$ for an ideal G-FET ${ }^{17}$ of

$$
N=\frac{W Q_{\mathrm{n}}}{q L} .
$$

\section{CONCLUSIONS}

This work discusses the function of polar ligands at the graphene interface and how their influence could push the device to become a more unipolar GFET. This effect is studied under different top-gate electric fields, and it is concluded that the mobility changes are inherent to a negative top potential. This unipolar behavior is achieved not only through an apparent suppression of the effective electron mobility but also by a large increase in the effective hole mobility.

\section{ACKNOWLEDGEMENTS}

This work was partially financially supported by the Knut and Alice Wallenberg Foundation (No. 2011.0082) and the Swedish Research Council (2014-5591).

\section{OPEN ACCESS}

This article is distributed under the terms of the Creative Commons Attribution 4.0 International License (http://creativecommons.org/licenses/by/4.0/), which permits unrestricted use, distribution, and reproduction in any medium, provided you give appropriate credit to the original author(s) and the source, provide a link to the Creative Commons license, and indicate if changes were made.

\section{REFERENCES}

1. K.S. Novoselov, A.K. Geim, S.V. Morozov, D. Jiang, Y. Zhang, S.V. Dubonos, I.V. Grigorieva, and A.A. Firsov, Science 306, 666-669 (2004).

2. J.H. Chen, C. Jang, S. Adam, M.S. Fuhrer, E.D. Williams, and M. Ishigami, Nat. Phys. 4, 377-381 (2008).

3. F. Schwierz, Nat. Nano 5, 487-496 (2010).

4. H. Wang, Y. Wu, C. Cong, J. Shang, and T. Yu, ACS Nano 4, 7221-7228 (2010).

5. M. Min, S. Seo, J. Lee, S.M. Lee, E. Hwang, and H. Lee, Chem. Commun. 49, 6289-6291 (2013).

6. A.K. Geim and K.S. Novoselov, Nat. Mater. 6, 183-191 (2007).

7. S.F. Chowdhury, L. Tao, S. Banerjee, D. Akinwande in Presented at the 14th IEEE International Conference on Nanotechnology, 2014 (unpublished).

8. P. Ahlberg, M. Hinnemo, M. Song, X. Gao, J. Olsson, S.-L. Zhang, and Z.-B. Zhang, Appl. Phys. Lett. 107, 203104 (2015).

9. P. David and L. Haitao, 2D Mater. 2, 032001 (2015).

10. S. Kim, J. Nah, I. Jo, D. Shahrjerdi, L. Colombo, Z. Yao, E. Tutuc, and S.K. Banerjee, Appl. Phys. Lett. 94, 062107 (2009).

11. M. Hinnemo, P. Ahlberg, C. Hägglund, W. Ren, H.-M. Cheng, S.-L. Zhang, and Z.-B. Zhang, Carbon 98, 567-571 (2016).

12. M.S. Fuhrer and J. Hone, Nat. Nano 8, 146-147 (2013).

13. R. Kotipalli, R. Delamare, O. Poncelet, X. Tang, L.A. Francis, and D. Flandre, EPJ Photovolt. 4, 45107 (2013).

14. G. Dingemans, M.C.M. van de Sanden, and W.M.M. Kessels, Electrochem. Solid-State Lett. 13, H76-H79 (2010).

15. C.M. Aguirre, P.L. Levesque, M. Paillet, F. Lapointe, B.C. St-Antoine, P. Desjardins, and R. Martel, Adv. Mater. 21, 3087-3091 (2009).

16. M. Lafkioti, B. Krauss, T. Lohmann, U. Zschieschang, H. Klauk, K.V. Klitzing, and J.H. Smet, Nano Lett. 10, 11491153 (2010).

17. T. Fang, A. Konar, H. Xing, and D. Jena, Appl. Phys. Lett. 91, 092109 (2007). 